\title{
MOVEMENT IN ACTIVE PRODUCTION NETWORKS
}

\author{
Mark A. Jones
}

Alan S. Driscoll

AT\&T Bell Laboratories

Murray Hill, New Jersey 07974

\begin{abstract}
We describe how movement is handled in a class of compulational devices called active production networks (APNs). The APN model is a parallel, activation-based framework that has been applied to other aspects of natural language processing. The model is briefly defined, the notation and mechanism for movement is explained, and then several examples are given which illustrate how various conditions on movement can naturally be explained in terms of limitations of the APN device.
\end{abstract}

\section{INTRODUCTION}

Movernent is an important phenomenon in natural languages. Recently, proposals such as Gazdar's derived rules (Gazdar, 1982) and Pereira's extraposition grammars (Pereira, 1983) have attempted to find minimal extensions to the context-free framework that would allow the description of movement. In this paper. we describe a class of computational devices for natural language processing. called active production metworks (APNs), and explore how certain kinds of movernent are handled. In particular. we are concerned with left extraposition. such as Subjectauxiliary Inversion. Wh-movement, and NP holes in relative clauses. In these cases, the extraposed constituent leaves a trace which is inserted at a later point in the processing. This paper builds on the research reported in Jones (1983) and Jones (forthcoming).

\section{ACTIVE PRODUCTION NETWORKS}

\section{The Derice}

Our contention is that only a class of parallel devices will prove to be powerful enough to allow broad contextual priming. to pursue alternative hypotheses, and to explain the paradox that the performance of a sequential system often degrades with new knowledge, whereas human performance usually improves with learning and experience.' There are a number of new parallel processing (connectionist) models which are sympathetic to this view-Anderson (1983). Feldman and Ballard (1982), Wallz and Pollack (1985). McClelland and Rumethart (1981, 1982), and Fahlman, Hinton and Sejnowski (1983).

Many of the connectionist modets use iterative relaxation techniques with networks containing excitatory and inhibitory links. They have primarily been used as best-fit categorizers in large recognition spaces, and it is not yet clear how they will implement the rule-governed behavior of parsers or problem solvers. Ruie-based systems need a strong notion of an operating state, and they depend heavily on appropriate variable binding sehemes for opera. tions such as matching (e.g.. unitication) and recursion. The APN model directly supports a rule-based interpreta. tion, while retaining much of the general flavor of

1. The bumen ability to pesform competetionally expensive operetions usion relecivaly alow, paralled bardware reinforces this belief. connectionism. An active production network is a ruleoriented, distributed processing system based on the following principles:

1. Each node in the network executes a uniform activation algorithm and assumes states in response to messages (such as expectation, inhibition, and activation) that arrive locally; the node can, in turn, relay messages, initiate messages, and spawn new instances to process message activity. Although the patterns that define a node's behavior may be quite idiosyncratic or specialized, the algorithm that interprets the pattern is the same for each node in the network.

2. Messages are relatively simple. They have an associated time, strength, and purpose (e.g.. to post an expectation). They do not encode complex structures such as entire binding lists, parse trees, feature lists, or meaning representations. ${ }^{2}$ Consequently, no structure is explicitly built; the "result" of a computation consists entirely of the activation trace and the new state of the network.

Figure 1 gives an artificial, but comprehensive exampic of an APN grammar in graphical form. The grammar generates the strings $-a, b$, acd, ace. $b c d, b c e, f g$ and $g-$ and illustrates many of the pattern language features and grammar writing paradigms. The network responds to sources which activate the network at its leaves. Activation messages spread "upward" through the network. At conjunctive nodes (seq and and). expectation messages are posted for the legal continuations of the pattern: inhibition messages are sent down previous links when new activations are recorded.

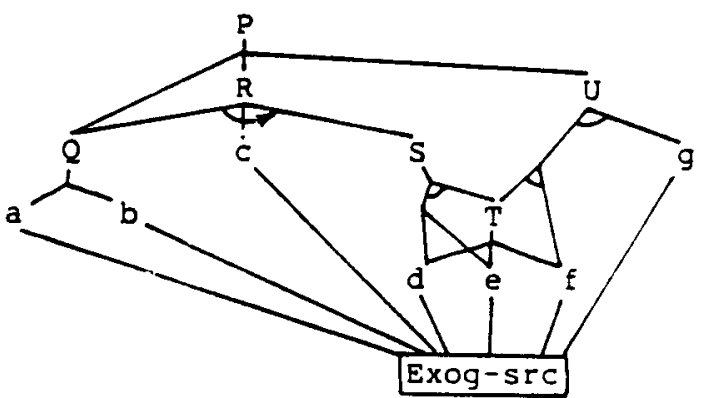

Figure 1. A Sample APN

In parsing applications. partially instantiated nodes are viewed as phrase structure rules whose next constituent is expected. The sources primarily arise from exogenous

2. For s simuinr connectioniut view, sen Feldman and Ballard (1982) or Waltz and Pollack (1985). A comperison of marker pasing. value

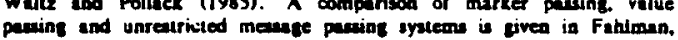

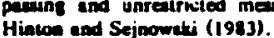


strobings of the network by external inputs. In generation or problem solving applications, partially instantiated nodes are viewed as partially satisfied goals which have outstanding subgoals whose solutions are desired. The sources in this case are endogenously generated. The compatibility of these two views not only allows the same network to be used for both parsing and generation, but also permits processes to share in the interaction of internal and external sources of information. This compatibility, somewhat surprisingly, turned out to be crucial to our treatment of movement, but it is also clearly desirable for other aspects of natural language processing in which parsing and problem solving interact (e.g., reference resolution and inference).

\section{The Patters Lascuge}

Each node in an APN is defined by a pattern, written in the pattern language of Figure 2. A pattern describes the messages to which a node responds. and the new messages and internal states that are produced. Each subpat. tern of the form ( $s$ binding-pat) in the pattern for node $N$ is a variable binding site; a variable binding takes place when an instance of a node in binding-pat activates a reference to variable $v$ of node $N$. Implicitly, a pattern defines the set of states and state transitions for a node. The ? (optionality). + (repetition) and - (optional repetition) operators do not extend the expressiveness of the language, but have been added for convenience. They can be replaced in preprocessing by equivalent expressions.' Formal semantic definitions of the measage passing behavior for each primitive operator have been specified.

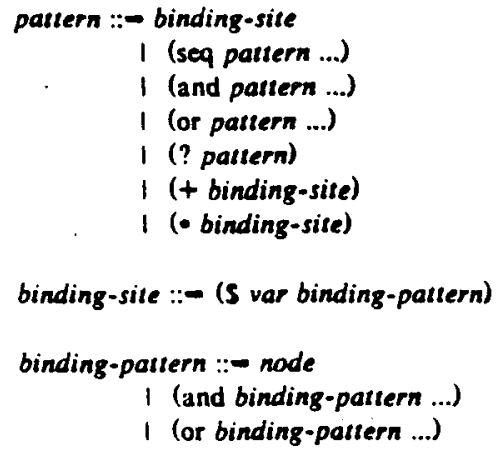

Figure 2. The APN Pattern Language

An important distinction that the pattern language makes is in the synchronicity ${ }^{4}$ of activation signals. The pattern (and $(S \vee / X)(S \vee 2 n)$ requires that the activation from $X$ and $Y$ emanate from distinct network sources, while the pattern ( $\$ \vee$ (and $X Y)$ ) insists that instances of $X$ and $Y$ are activated from the same source. In the

3. The exect cbouce of operaton is the pattern lenguage is a conew bat separale irewe from the specticalion of tbe APN mechipe.

4. The curreat APN model sllocale souroes requentially. The term synchromicity reflocts the fact that the hource identity of two ectivation mennges cen be locally computied freen their time of arrivel. This worts 42 lons as tbe ectivetion proces mas fese esough to coodition the network

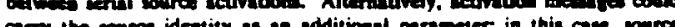

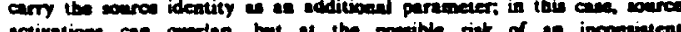

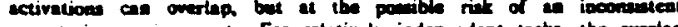

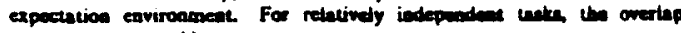
way noe pow a problem. graphical representation of an APN, synchrony is indicated by a short tail above the subpattern expression: the definition of $U$ in Figure 1 illustrates both conventions: (and $(S \vee l$ (and $T \rho)(S \vee 2 g)$ ).

\section{As Erangin}

Figure 3 shows the stages in parsing the string acd. An exogenous source Exog-srco first activates $a$, which is not currently supported by a source and, hence, is in an inactive state. The activation of an inactive or inhibited node gives rise to a new instance $(a 0)$ to record the binding. The instance is effectively a new node in the network, and derives its pattern from the spawning node. The activation spreads upward to the other instances shown in Figure 3(a). The labels on each node indicate the current activation level, represented as an integer between 0 and 9, inclusive.

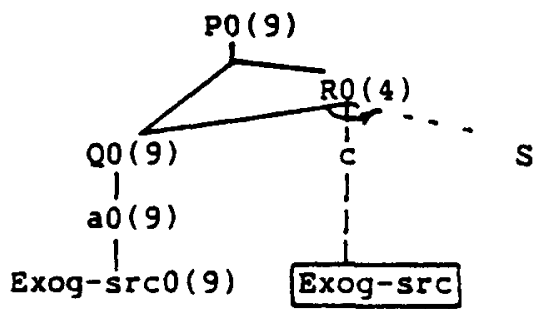

(a) trace structure after a

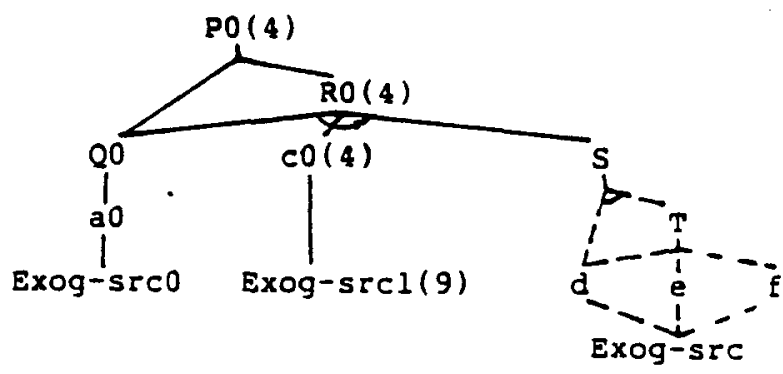

(b) trace structure after ac

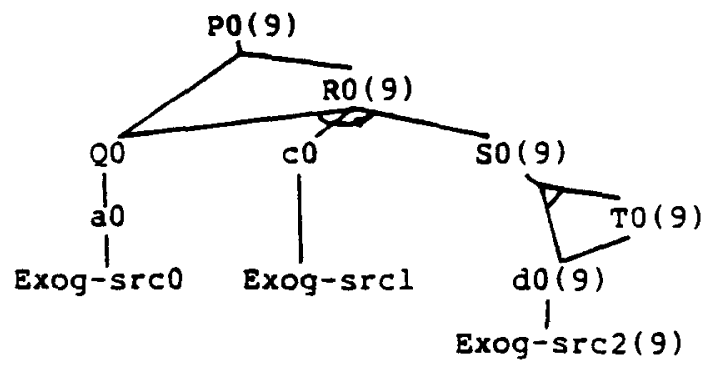

(c) trace structure after acd

Foure 3. Stages in Parsing acd 
The activation of a node causes its pattern to be (re)instantiated and a variable to be (re) bound. For example, in the activation of $R O$, the pattern (seq $(S v l Q$ ) (S $v 2 C)$ is replaced by (seq (S v/ (or $Q Q 0)$ ) (S v2 C)), and the variable $v /$ is bound to $Q O$. For simplicity, only the active links are shown in Figure 3. RO posts an expectation measage for node $C$ which can further its pattern. The source Exog-srco is said to be supporting the activation of nodes a0, QO, RO and $P O$ above it, and the expectations or inhibitions that are generated by these nodes. For the current paper we will assume that exogenous sources remain fully on for the duration of the sentence."

In Figure 3(b), another exogenous source Exog-srcl activates $c$, which furthers the pattern for $R O$. RO sends an inhibition message to $Q O$, posts expectations for $S$, and relays an activation message to $P O$. which rebinds its variable to $R O$ and assumes a new activation value. Figure 3(c) shows the final situation after $d$ has been activated. The synchronous conjunction of $S O$ is satisfied by $T O$ and do. $R O$ is fully satisfied (activation value of 9 ), and $P O$ is re-satisfied.

\section{Granarer Writine Paradigum}

The APN in Figure 1 illustrates several grammar writing paradigms. The situation in which an initial prefix string ( $a$ or $b$ ) satisfies a constituent $(P)$, but can be foilowed by optional suffix strings ( $c d$ or $c e$ ) occurs frequently in natural language grammars. For example, noun phrase heads in English have optional prenominal and postnominal modifiers. The synchronous disjunction at $P$ allows the local role of $a$ or $b$ to change, while preserving its interpretation as part of a $P$. It is aiso simple to encode optional prefixes.

Another common situation in natural language grammars is specialization of a constituent based on some internal feature. Noun phrases in English, for example, can be specialized by case; verb phrases can be specialized as participial, tensed or infinitive. In Figure 1 , node $S$ is a specialization which represents " $T_{s}$ with $d$-ness or e-ness, but not $f$-ness." The specialization is cunstructed by a synclironous conjunction of features that arise from subtrees sorne. where below the node to be specialized.

The APN model also provides for node outputs to be partitioned into independent classes for the purposes of the activation algorithm. The nodes in the classes form levels in the network and represent orthogonal systems of classification. The cascading of expectutions from different levels can implement cuntext-sensitive behaviors such as feature agreement and scmantic sclectional restrictions. This is described in Jones (forthcoming). In the next section, we will introduce a grammar writing paradigm to represent movement. another type of nun-context-free behavior.

S. It is interesting to speculate on the consequencea of varivene reisxations of this seumption. Fundementat limilations in the allocation of wurce may be relatod to limilations in sburt term memory (or buffer apace is determiaistic mudeb: Marcus, 1980). Linzuintic corvernint based on concituent length could be rehated to cource decay. Som ayntactic

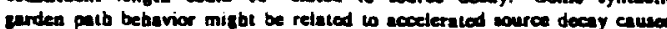
by intibition from a competing bypotbecia Anything more than a foocone is premature at this time.

\section{MOVEMENT}

From the APN perspective, movement (limited here to left-extraposition) necessitates the endogenous reactivation of a trace that was created earlier in the process. To capture the trace so that expectations for its reactivation can be posted, we use the following type of rule: (seq (S $v 1$... $X \ldots)(s \vee 2 \ldots$ (and $X X$-sre $\eta$...). When an instance, $X O$, first activates this rule, $v /$ is bound to $X 0$; the second occurrence $X$ in the rule is constrained to match instances of $X O$, and expectations for $X O, X$-src and $Y$ are created. No new exogenous source can satisfy the synchronous conjunction: oniy an endogenous $X$-sre can. The rule is similar to the notion of an $X$ followed by a $Y$ with an $X$ hole in it (cf. Gazdar, 1982).

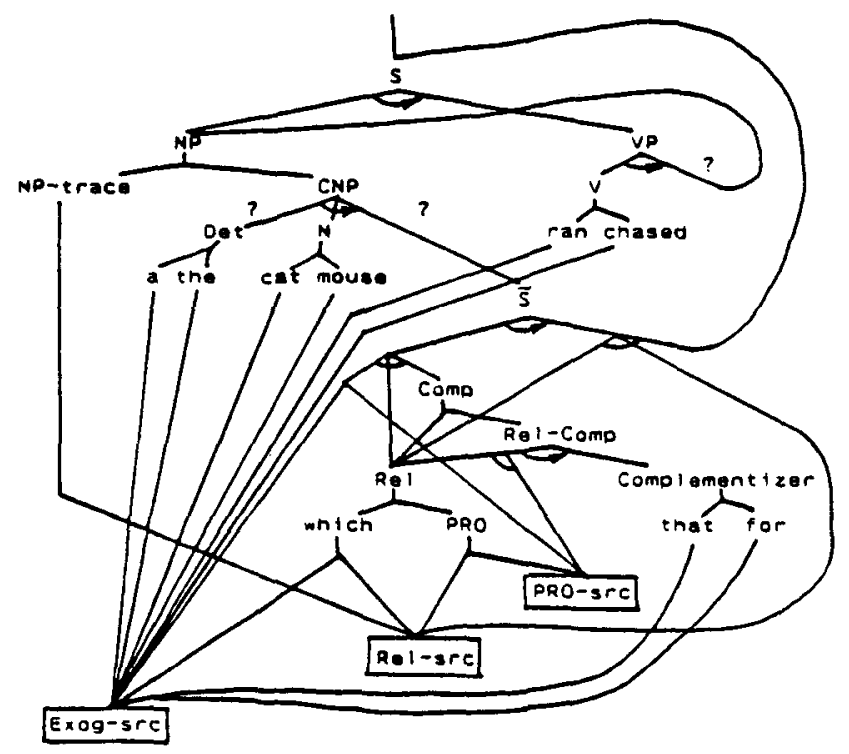

Figure 4. A Grammar for Relative Clauses

Figure 4 defines a grainmar with an NP hole in a rela. live clausc: other types of left-extraposition are handled analoguusly. Our treatment of relatives is adapted from Chumsky and Lasnik (1977). The movement ruie for $\bar{S}$ is: (seq (S v/ (and Comp Rel (or Exog-src PRO-sre)) (s v2 (and Ret Rel-sre $S$ ))). The rule restricts the first instance of $R e l$ to arise either from an exogenous relative pronoun such as which or from an endogenously generated (phonologically null) pronoun $P R O$. The second variable is satisfied when Rel-sre simultaneously reactivates a trace of the Rel instance and inserts an NP-trace into an $S$.

It is instructive to consider how phonologically null pronouns are inserted before we discuss how movement occurs by trace insertion. The phrase, $\left[_{N P}\right.$ the mouse $[\bar{S}$ PRO; that ...ll, illustrates how a relative pronoun $P R O$ is inserted. Figure $5(a)$ shows the network after parsing the cat. When the complementizer that appears next in the input. $P R O$-src receives inhibition (marked by downward arrows in Figure $5(b))$ from Rel-Compo. Non-exogenous 
sources such as $P R O$-sre and Rel-sre are activated in contexts in which they are expected and then receive inhibition. Figure S(c) shows the resulting network after PROsre has been activated. The inserted pronoun behaves precisely as an input pronoun with respect to subsequent movement.

The trace generation necessary for movement uses the same insertion mechanism described above. Figures 6(a)(d) illustrate various stages in parsing the phrase. [NP the cat [ $s$ whichi $\left[s t_{i}\right.$ ranl]]. In Figure 6(a), after parsing the cat which, synchronous expectations are posted for an $S$ which contains a reactivation of the RelO trace by Relsre. The signal sent to $S$ by Rel-sre will be in the form of an NP (through NP-trace).

Figure 6(b) shows how the input of ran produces inhibition on Rel-sre from SI. The inhibition on Rel-sre causes it to activate (just as in the null pronoun insertion) to try to satisfy the current contextual expectations. Figure 6 (c) shows the network after Rel-src has activated to supply the trace. The only remaining problem is that Rel-sre is actively inhibiting itself through $\overline{S 0} .6$ When Rel-sre activates again, new instances are created for the inhibited nodes as they are re-activated; the uninhibited nodes are simply rebound. The final structure is shown in Figure 6(d).

It is interesting that the network automatically enforces the restriction that the relative pronoun, complementizer and subject of the embedded sentence cannot all be missing. PRO must be generated before its trace can be inserted as the subject. Furthermore. since expectations are strongest for the first link of a sequence, expectations will be much weaker for the $V P$ in the relative clause (under $S$ under $S$ ) than for the top-level VP under $S O$.

The fact that the device blocks certain structures, without explicit well-formedness constraints, is quite significant. Wherever possible. we would like to account for the complexity of the data through the composite behavior of a universal device and a simple, general gram. mar. We consider the description of a device which embodies the appropriate principles more parsimonious than a list of complex conditions and filters, and. to the extent that its architecture is independently motivated by processing (i.e., performance) considerations, of greater theorctical interest.'

As we have seen, certain interpretations can be suppressed by expectations from elsewhere in the network. Furthermore, the occurrence of traces and empty constituents is severely constrained because they must be supplied by endugenous sources. which can only suppurt a single constituent at any given time. For NP movement, these two properties of the device, taken together, etfectively enforce Ross's Complex NP Constraint (Ross, 1967), which states that, "No element contained in a

6. Anotber way of uating this is that the nop-synchronicity of the 1 mo variables in the pattern bas been vidated. The velf-inbibition of a source cocurn in aber contexts in the APN iramework, evep for exosenow cocurn is aber contexts in the APN Iramework, even for exogenow

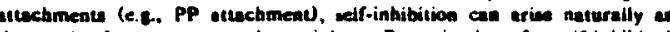

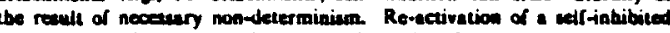

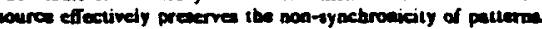

7. The wort of Marcese (1980) is in thin same spirit. sentence dominated by an NP with a lexical head noun may be moved out of that NP by a transformation."

To see why this constraint is enforced, consider the two kinds of sentences that an NP with a lexical head noun might dominate. If the embedded sentence is a relative clause, as in, INP the rat $l_{s}$ which, $l_{s}$ the cat $I_{s}$ whichj [s $t_{\text {f }}$ chased $t_{i}$ l] likes fishll], then Rel-sre cannot support both traces. If the embedded sentence is a noun complement (not shown in Figure 4), as in, INP $_{N}$ the rat l $_{\vec{S}}$ which, Is he read a report Is that is the cat chased $\left.\left.t_{[}\right][\}\right]$. then there is only one trace in the intended interpretation, but there is nondeterminism during parsing between the noun complement and the relative clause interpretation. The interference causes the trace to be bound to the innermost relative pronoun in the relative clause interpretation." Thus, the combined properties of the device and grammar consistently block those structures which violate the Complex NP Constraint. Our preliminary findings for other types of movement (e.g., Subjectauxiliary (nversion, Wh-movement, and Raising) indicate that they also have natural APN explanations.

\section{IMPLEMENTATION and FUTURE DIRSCTIONS}

Although the research described in this summary is primarily of a theoretic nature. the basic ideas involved in using APNs for recognition and generation are being implemented and tested in Zetalisp on a Symbolics Lisp Machine. We have also hand-simulated data on movement from the literature to design the theory and algorithms presented in this paper. We are currently designing networks for a broad coverage syntactic grammar of English and for additional, caseaded levels for NP role mapping and case frames. The model has also been adapted as a general, context-driven problem solver, although more work remains to be done.

We are considering ways of integrating iterative retaxation techniques with the rule-based framework of APNs. This is particularly necessary in helping the network to identify expectation coalitions. In Figure $5(a)$, for example. there should be virtually no expectations for Rel-sre. since it cannot satisfy any of the dominating synchronous conjunctions. Some type of non-activating feedback from the sources seems to be necessary.

\section{SUMMARY}

Recent linguistic theories have attempted to induce general principles (e.g., CNPC. Subjacency, and the Structure Preserving Hypothesis) from the detailed structural descriptions of earlier transformational theories (Chomsky, 1981). Our research can be viewed as an attempt to induce the machine that embodies these principles. In this paper, we have described a class of candidate machines, called active production networks, and outlined how they handle movement as a natural way in which machine and grammar interact.

The APN framework was initially developed as a plausible cognitive model for language processing. which would have real-time processing behavior. and extensive

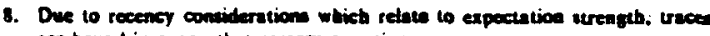
are bound in a way thet preserves netial. 


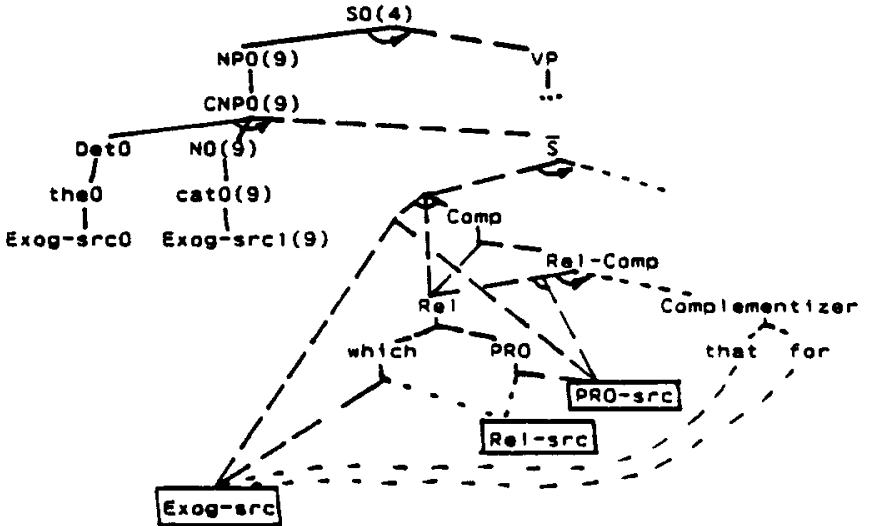

(a) trace structure after the cat

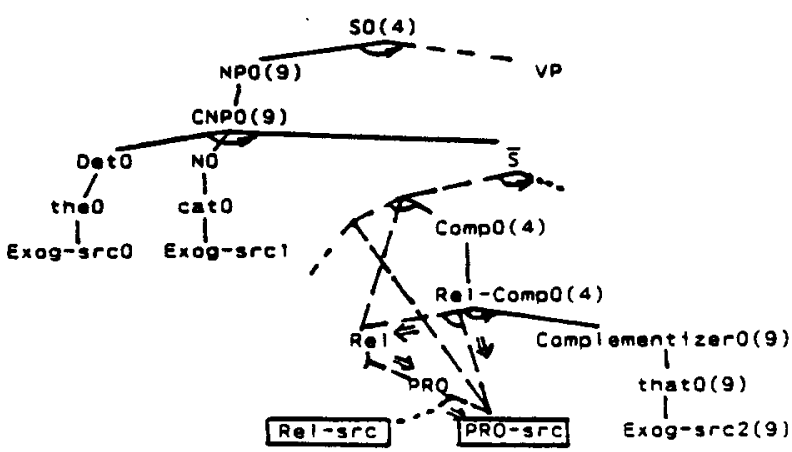

(b) trace structure after the cat ... thot

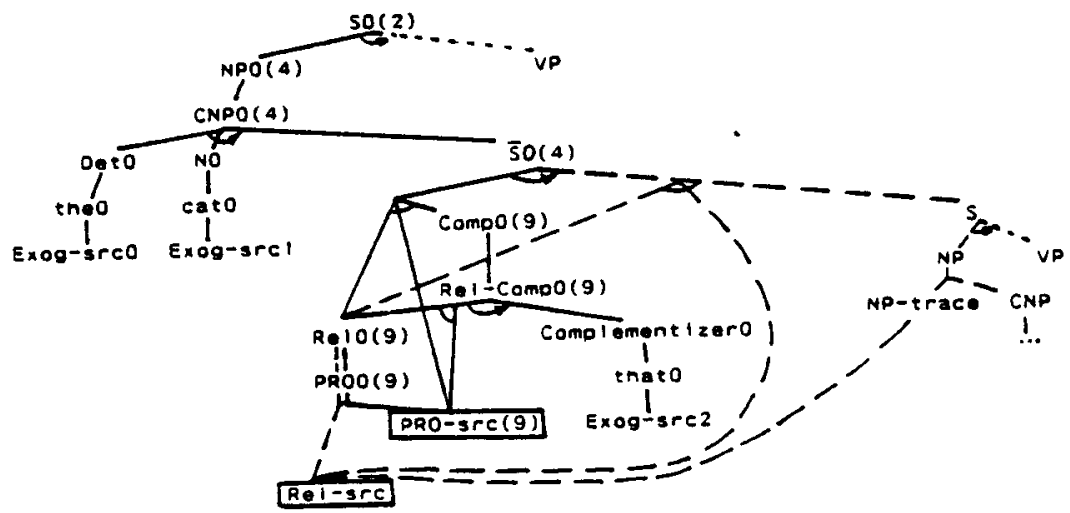

(c) trace structure after the cat PRO that

Figure 5. Relative Pronoun Insertion

contextual processing and learning capabilities based on a formal notion of expectations. That muventent also seems naturally expressible in a way that is consistent with current linguistic theories is quite intriguing.

\section{REFERENCES}

Anderson, J. R. (1983). The Archilecture of Cognition, Harvard University Press, Cambridge.

Chomsky, N. (1981). Lectures on Government and Binding. Foris Publications, Dordrecht.

Chomsky, N. and Lasnik, H. (1977). "Filters and Control," Linguistic Inquiry 8, 425-504.

Fahlman, S. E. (1979). NETL: A System for Representing and Using Real-World Knowledge, MIT Press, Cambridge.

Fahlman, S. E., Hinton, G. E. and Sejnowski, T. J. (1983). "Massively Parallel Architectures for Al: NETL. Thistle, and Boltzmann Machines." AAAl-83 Conference Proceedings.

Feldman, J. A. and Ballard, D. H. (1982). "Cunnectionist Models and Their Properties," Cognitive Science 6, 205-254.
Gazdar, G. (1982). "Phrase Structure Grammar," The Nature of Syntactic Representation. Jacubson and Pullum. eds., Reidel, Boston, 131-186.

Jones. M. A. (1983). "Activation-Based Parsing," 8th IJCAI, Karisruhe, W. Germany, 678-682.

Jones, M. A. (forthcoming). submitted for publication.

Marcus, M. P. (1980). A Theory of Syntactic Recognition for Natural Lunguage. MIT Press, Cambridge.

Pereira, F. (1983). "Logic for Natural Language Analysis," technical report 275. SRI International. Menio Park.

Ross, J. R. (1967). Constraints on Variables in Syntax, unpublished Ph.D. thesis, MIT, Cambridge.

Waltz, D. L. and Pollack, J. B. (1985). “Massively Parallel Parsing: A Strongly Interactive Model of Natural Language Interpresation," Cognitive Science, 9, 51-74. 

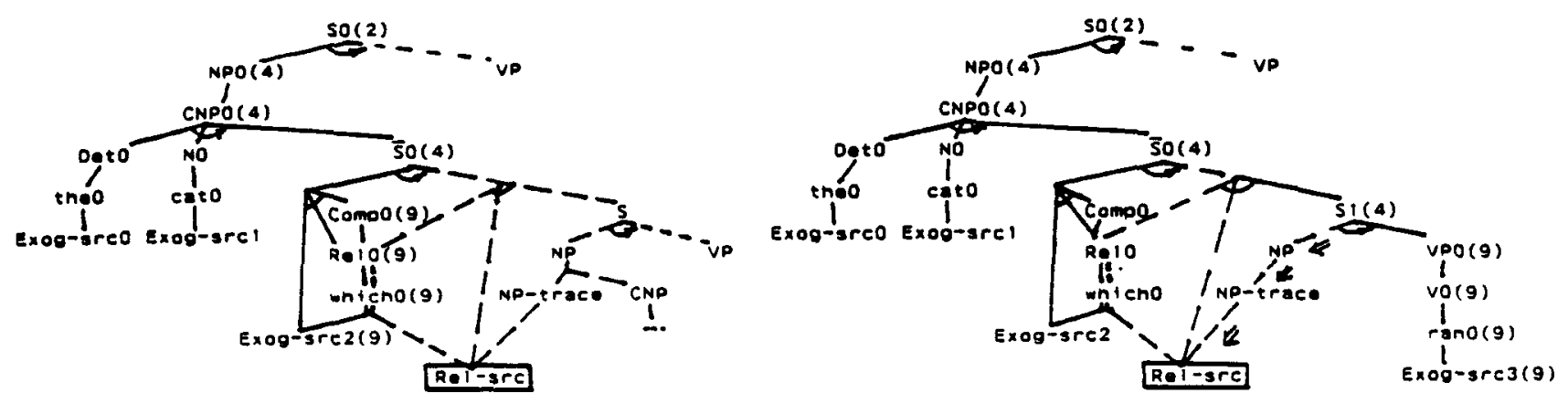

(a) trace structure after the cat which

(b) trace structure after the cat which ... ran
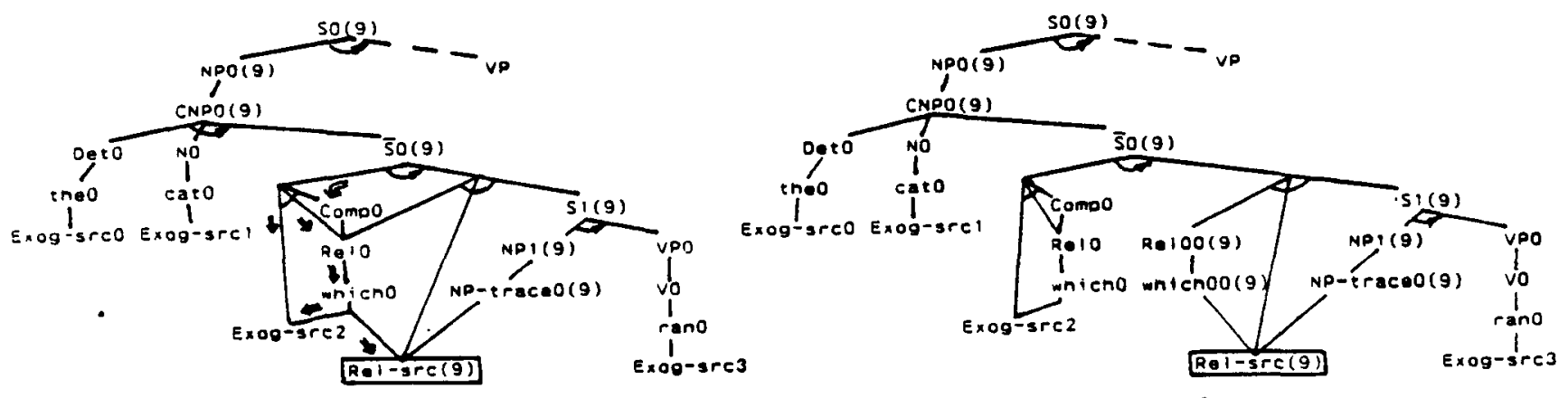

(c) trace structure just after the cat which t ran

(d) final trace structure

Figure 6. Parsing Relative Clauses 